\title{
Influence of electric current on the Casimir forces between graphene sheets
}

\author{
A.I.Volokitin ${ }^{1,2 *}$ and B.N.J.Persson ${ }^{1}$ \\ ${ }^{1}$ Peter Grünberg Institut, Forschungszentrum Jülich, D-52425, Germany and \\ ${ }^{2}$ Samara State Technical University, 443100 Samara, Russia
}

\begin{abstract}
We investigate the dependence of the thermal Casimir force and the Casimir friction force between two graphene sheets on the drift velocity of the electrons in one graphene sheet. We show that the drift motion produces a measurable change of the thermal Casimir force due to the Doppler effect. The thermal Casimir force as well as the Casimir friction are strongly enhanced in the case of resonant photon tunneling when the energy of the emitted photon coincides with the energy of electron-hole pair excitations. In the case of resonant photon tunneling, even for temperatures above room temperature the Casimir friction is dominated by quantum friction due to quantum fluctuations. Quantum friction can be detected in frictional drag experiment between graphene sheets for high electric field.
\end{abstract}

PACS: 42.50.Lc, 12.20.Ds, 78.67.-n

In the late 1940s Hendrik Casimir predicted $\underline{\underline{1}}$ that two macroscopic non-magnetic bodies with no net electric charge (or charge moments) can experience an attractive force much stronger than gravity. The existence of this force is one of the few direct macroscopic manifestations of quantum mechanics; others are superfluidity, superconductivity, and the black body radiation spectrum.

Casimir based his prediction on a simplified model involving two parallel perfectly conducting plates separated by vacuum. A theory of the van der Waals and Casimir forces between parallel material plates in thermal equilibrium and separated by a vacuum gap was developed by Lifshitz (1955) $)^{2}$. Lifshitz's theory describes dispersion forces between dissipative media as a physical phenomenon caused by the fluctuating electromagnetic field that is always present in both the interior and the exterior of any medium. Outside the medium this field exists partly in the form of the radiative propagating waves, and partly in the form of nonradiative evanescent waves whose amplitudes decay exponentially with the distance away from the medium. To calculate the fluctuating electromagnetic field Lifshitz used Rytov's theory $\underline{\underline{3}-\underline{5}}$. Rytov's theory is based on the introduction into the Maxwell equation of a "random" field (just as, for example, one introduces a "random" force in the theory of Brownian motion). Both quantum and thermal fluctuations give contributions to the total Casimir force. A general theory of Casimir and van der Waals forces was developed in Ref $\underline{\underline{6}}$ using quantum field theory. This theory confirmed the results of Lifshitz's theory. Quantum fluctuations dominate at small separation $\left(d<\lambda_{T}=c \hbar / k_{B} T\right)$ and thermal fluctuations dominate at large separation $\left(d>\lambda_{T}\right)$. Casimir forces due to quantum fluctuations have been studied experimentally for a long time $\mathrm{e}^{7,8}$. However the Casimir forces due to thermal fluctuations were measured only recently, and the results confirmed the prediction of the Lifshitz theory ${ }^{9}$. At present the interest in Casimir forces is increasing because they dominate the interaction between nanostructures and are responsible for the adhesion between moving parts in small devices such as micro- and nano-electromechanical systems $\underline{10,11}$. Due to this practical interest and the fast progress in force detection techniques, experimenta ${ }^{9,12}-15$ and theoretical ${ }^{16,17}$ investigations of Casimir forces have experienced an extraordinary "renaissance" in the past few years. At present a great deal of attention is devoted to the study of the Casimir forces in graphene systems $18-26$.

Graphene, isolated monolayer of carbon, which was obtained very recently, consists of carbon atoms densely packed into a two-dimensional honeycomb crystal lattice. The unique electronic and mechanical properties of graphene are actively studied both theoretically, and experimentally because of their importance for fundamental physics, and also possible technological applications $\underline{27}$ - $\underline{30}$. In particular, the valence band and conduction band in graphene touch each other at one point named the Dirac point. Near this point the energy spectrum for electrons and holes has a linear dispersion. Due to this linear (or "conical") dispersion relation electrons and holes near this point behave like relativistic particles described by the Dirac equation for massless fermions. Because of the unusual electronic properties of graphene, the Casimir forces in graphene also have unusual properties. Contribution to the Casimir force due to thermal fluctuations for normal materials dominates for $d>\gamma_{T}=\hbar c / k_{B} T$ but for two graphene sheets thermal contribution dominates for much shorter distances 26 s $d>\xi_{T}=\hbar v_{F} / k_{B} T$, where $v_{F} \sim 10^{6} \mathrm{~m} / \mathrm{s}$ is the Fermi velocity in graphene. At room temperature the parameters $\xi_{T}$ and $\gamma_{T}$ are $25 \mathrm{~nm}$ and $7.6 \mu \mathrm{m}$, respectively. This property makes it possible to measure the thermal Casimir force using an atomic force microscope or other force

\footnotetext{
* Corresponding author. E-mail address:alevolokitin@yandex.ru
} 
measuring techniques. Tailoring the thermal Casimir force using Fermi level tuning by gate voltage was discussed in Ref. 24.

Alternative method of tailoring the thermal Casimir force consists in driving an electric current in a graphene sheet. It was shown by Pendry $\underline{31}$ that the reflection amplitudes from moving metal surface are modified due to the Doppler effect. The same modification of reflection amplitudes can be obtained if instead of motion of metal plate, a drift motion of charge carriers is induced in it by applied voltage ${ }^{32}$. Due to the high mobility of carriers in graphene, in a high electric field electrons (or holes) can move with very high velocities (up to $10^{6} \mathrm{~m} / \mathrm{s}$ ). The drift motion of charge carries in graphene will result in a modification of dielectric properties (and the Casimir force) of graphene due to the Doppler effect ${ }^{31}$. If in one of two parallel graphene sheets an electric current is induced, then the electromagnetic waves, radiated by the graphene sheet without an electric current, will experience a frequency Doppler shift in the reference frame moving with the drift velocity $v$ of electrons in the other graphene sheet: $\omega^{\prime}=\omega+q_{x} v$, where $q_{x}$ is the parallel to the surface component of momentum transfer. The same is true for the waves emitted by the other graphene sheet. Due to the frequency dependence of the reflection amplitudes the electromagnetic waves will reflect differently in comparison to the case when there is no drift motion of electrons, and this will give rise to the change of the Casimir force.

In this Letter, we investigate the dependence of the thermal Casimir force between graphene sheets on the drift velocity $v$ of charge carriers in one of the graphene sheet. Let us consider two graphene sheets separated by vacuum gap with thickness $d \ll \lambda_{T}=c \hbar / k_{B} T$. Assume that the free charge carriers in one graphene sheet move with drift velocity $v \ll c$ along the $x$-axis ( $c$ is the light velocity) relative to the other graphene sheet. Because a drift motion of the free charge carriers produces a similar modification of the reflection amplitudes as in the case of moving graphene sheet, the theory of the Casimir forces between moving bodies ${ }^{33}$ can be used to calculate the Casimir forces between sheets (both of which are at the rest) in presence of the drift motion of the free charge carriers in one graphene sheet. The force which acts on the surface of the sheet can be calculated from the Maxwell stress tensor $\sigma_{i j}$, evaluated at the surface of the sheet at $z=0$ :

$$
\begin{gathered}
\sigma_{i j}=\frac{1}{4 \pi} \int_{0}^{\infty} d \omega \int \frac{d^{2} q}{(2 \pi)^{2}}\left[<E_{i} E_{j}^{*}>+<E_{i}^{*} E_{j}>+<B_{i} B_{j}^{*}>+<B_{i}^{*} B_{j}>\right. \\
\left.-\delta_{i j}\left(<\mathbf{E} \cdot \mathbf{E}^{*}>+<\mathbf{B} \cdot \mathbf{B}^{*}>\right)\right]_{z=0}
\end{gathered}
$$

where $\langle\ldots\rangle$ denotes statistical average over the random the electric $\mathbf{E}$ and magnetic induction $\mathbf{B}$ field. According to Ref $\underline{33}$ the Casimir force $F_{z}=\sigma_{z z}$ between moving media is determined by

$$
F_{z}=F_{z T}+F_{z 0},
$$

where the temperature dependent term $F_{z T}$ and the zero-temperature contribution $F_{z 0}$ are given by

$$
\begin{gathered}
F_{z T}=\frac{\hbar}{\pi^{3}} \int_{0}^{\infty} d q_{y} \int_{0}^{\infty} d q_{x} q e^{-2 q d}\left\{\int _ { 0 } ^ { \infty } d \omega \left(\frac{\operatorname{Im} R_{1}(\omega) \operatorname{Re} R_{2}\left(\omega^{+}\right) n_{1}(\omega)+\operatorname{Re} R_{1}(\omega) \operatorname{Im} R_{2}\left(\omega^{+}\right) n_{2}\left(\omega^{+}\right)}{\left|1-e^{-2 q d} R_{1}(\omega) R_{2}\left(\omega^{+}\right)\right|^{2}}\right.\right. \\
\left.+(1 \leftrightarrow 2))+\int_{0}^{q_{x} v} d \omega\left(\frac{\operatorname{Re} R_{1}\left(\omega^{-}\right) \operatorname{Im} R_{2}(\omega) n_{2}(\omega)}{\left|1-e^{-2 q d} R_{1}\left(\omega^{-}\right) R_{2}(\omega)\right|^{2}}+(1 \leftrightarrow 2)\right)\right\}, \\
F_{z 0}=\frac{\hbar}{2 \pi^{3}} \int_{0}^{\infty} d q_{y} \int_{0}^{\infty} d q_{x}\left\{\operatorname { R e } \int _ { 0 } ^ { \infty } d \omega s e ^ { - 2 s d } \left(\frac{R_{1}(i \omega) R_{2}\left(i \omega+q_{x} v\right)}{1-e^{-2 s d} R_{1}(i \omega) R_{2}\left(i \omega+q_{x} v\right)}\right.\right. \\
\left.+(1 \leftrightarrow 2))+\int_{0}^{q_{x} v} d \omega q e^{-2 q d}\left(\frac{\operatorname{Im} R_{1}(\omega) \operatorname{Re} R_{2}\left(\omega^{-}\right)}{\left|1-e^{-2 q d} R_{1}(i \omega) R_{2}\left(\omega^{-}\right)\right|^{2}}+(1 \leftrightarrow 2)\right)\right\},
\end{gathered}
$$

where $n_{i}(\omega)=\left[\exp \left(\hbar \omega / k_{B} T_{i}\right)-1\right]^{-1}(i=1,2), q=\sqrt{q_{x}^{2}+q_{y}^{2}}, s=\sqrt{(\omega / c)^{2}+q^{2}}, T_{i}$ is the temperature of $i$-th graphene sheet, $R_{i}$ is the reflection amplitude for surface $i$ for $p$-polarized electromagnetic waves, and $\omega^{ \pm}=\omega \pm q_{x} v$. 
The symbol $(1 \leftrightarrow 2)$ denotes the terms that are obtained from the preceding terms by permutation of 1 and 2 . In the first term in Eq. (4) the integration along the real axis was transformed into integration along the imaginary axis.

The reflection amplitude for a $2 \mathrm{D}$-system is determined by 34

$$
R_{i}=\frac{\epsilon_{i}-1}{\epsilon_{i}+1}, \epsilon_{i}=\frac{4 \pi p \sigma_{i}}{\omega \varepsilon}+1
$$

where $p=\sqrt{(\omega / c)^{2}-q^{2}}, \sigma_{i}$ is the longitudinal conductivity of the sheet which can written in the form $\sigma_{i}=-i \omega e^{2} \Pi_{i}(\omega, q) / q^{2}$ where $\Pi_{i}$ is the $2 \mathrm{D}$ polarizability. The dielectric function of the sheet is determined by $\varepsilon_{i}(\omega, q)=1+v_{q} \Pi_{i}(\omega, q), v_{q}=2 \pi e^{2} / q$ is the $2 \mathrm{D}$ Coulomb interaction. In term of $\varepsilon_{i}$ the reflection amplitude can be written as

$$
R_{i}=\frac{p\left(\varepsilon_{i}-1\right)}{p\left(\varepsilon_{i}-1\right)+i q}
$$

In the integration on the real axis $p \approx i q$ for $d<\lambda_{T}$. Thus, in this case

$$
R_{i} \approx \frac{\varepsilon_{i}-1}{\varepsilon_{i}}
$$

On the imaginary axis $p=i s$. In the finite lifetime generalization according to the Mermin approximation ${ }^{35}$ the dielectric function is determined by

$$
\varepsilon(\omega, q) \approx 1+\frac{(\omega+i \gamma)\left(\varepsilon_{0}(\omega+i \gamma, q)-1\right)}{\omega+i \gamma\left(\varepsilon_{0}(\omega+i \gamma, q)-1\right) /\left(\varepsilon_{0}(0, q)-1\right)}
$$

where $\varepsilon_{0}(\omega, q)$ is the RPA dielectric function and $\gamma$ is the damping parameter. In the study below we used the dielectric function of graphene, which was calculated recently within the random-phase approximation (RPA) 36,37 . The small (and constant) value of the graphene Wigner-Seitz radius $r_{s}$ indicates that it is a weakly interacting system for all carries densities, making the RPA an excellent approximation for graphene (RPA is asymptotically exact in the $r_{s} \ll 1$ limit). The dielectric function is an analytical function in the upper half-space of the complex $\omega$-plane:

$$
\varepsilon_{0}(\omega, q)=1+\frac{4 k_{F} e^{2}}{\hbar v_{F} q}-\frac{e^{2} q}{2 \hbar \sqrt{\omega^{2}-v_{F}^{2} q^{2}}}\left\{G\left(\frac{\omega+2 v_{F} k_{F}}{v_{F} q}\right)-G\left(\frac{\omega-2 v_{F} k_{F}}{v_{F} q}\right)-i \pi\right\},
$$

where

$$
G(x)=x \sqrt{x^{2}-1}-\ln \left(x+\sqrt{x^{2}-1}\right),
$$

where the Fermi wave vector $k_{F}=(\pi n)^{1 / 2}, n$ is the concentration of charge carriers, the Fermi energy $\epsilon_{F}=\hbar v_{F} k_{F}$, $v_{F} \approx 10^{6} \mathrm{~m} / \mathrm{s}$ is the Fermi velocity. The damping parameter $\gamma$ is due to scattering against impurities and acoustic phonons in graphene sheet, and can be expressed through the low field mobility $\mu$ : $\gamma=e v_{F} /\left(\hbar k_{F} \mu\right)$. Scattering of the graphene carries by the acoustic phonons of graphene places an intrinsic limits on the low-field room temperature $\left(T_{0}=300 \mathrm{~K}\right)$ mobility, given by $\mu_{0}=20 \mathrm{~m}^{2} / \mathrm{Vs}$ at the graphene carriers density $10^{16} \mathrm{~m}^{-2}$ (see Ref ${ }^{38}$ ), which gives $\gamma=8 \cdot 10^{11} \mathrm{~s}^{-1}$. At other temperatures the mobility can be obtained using the relation $\mu=\mu_{0} T_{0} / T$.

In addition to the intrinsic friction due to scattering against impurities and phonons, on the electrons moving in the graphene sheet acts the extrinsic friction due to the interaction with electrons in the nearby graphene sheet. According to the theory of the Casimir friction ${ }^{33}$, the friction force $F_{x}=\sigma_{x z}=F_{x T}+F_{x 0}$, where at $d \ll \lambda_{T}$ and $v \ll c$ the contributions from thermal $\left(F_{x T}\right)$ and quantum $\left(F_{x 0}\right)$ fluctuations are given by ${ }^{31,39}-41$

$$
\begin{gathered}
F_{x T}=\frac{\hbar}{\pi^{3}} \int_{0}^{\infty} d q_{y} \int_{0}^{\infty} d q_{x} q_{x} e^{-2 q d}\left\{\int_{0}^{\infty} d \omega\left(\frac{\operatorname{Im} R_{1}(\omega) \operatorname{Im} R_{2}\left(\omega^{+}\right)}{\left|1-e^{-2 q d} R_{1}(\omega) R_{2}\left(\omega^{+}\right)\right|^{2}} \times\left[n_{1}(\omega)-n_{2}\left(\omega^{+}\right)\right]+(1 \leftrightarrow 2)\right)\right. \\
\left.\quad-\int_{0}^{q_{x} v} d \omega\left(\frac{\operatorname{Im} R_{1}(\omega) \operatorname{Im} R_{2}\left(\omega^{-}\right)}{\left|1-e^{-2 q d} R_{1}(\omega) R_{2}\left(\omega^{-}\right)\right|^{2}} n_{1}(\omega)+(1 \leftrightarrow 2)\right)\right\}, \\
F_{x 0}=-\frac{\hbar}{2 \pi^{3}} \int_{0}^{\infty} d q_{y} \int_{0}^{\infty} d q_{x} q_{x} e^{-2 q d} \int_{0}^{q_{x} v} d \omega\left(\frac{\operatorname{Im} R_{1}(\omega) \operatorname{Im} R_{2}\left(\omega^{-}\right)}{\left|1-e^{-2 q d} R_{1}(\omega) R_{2}\left(\omega^{-}\right)\right|^{2}}+(1 \leftrightarrow 2)\right)
\end{gathered}
$$



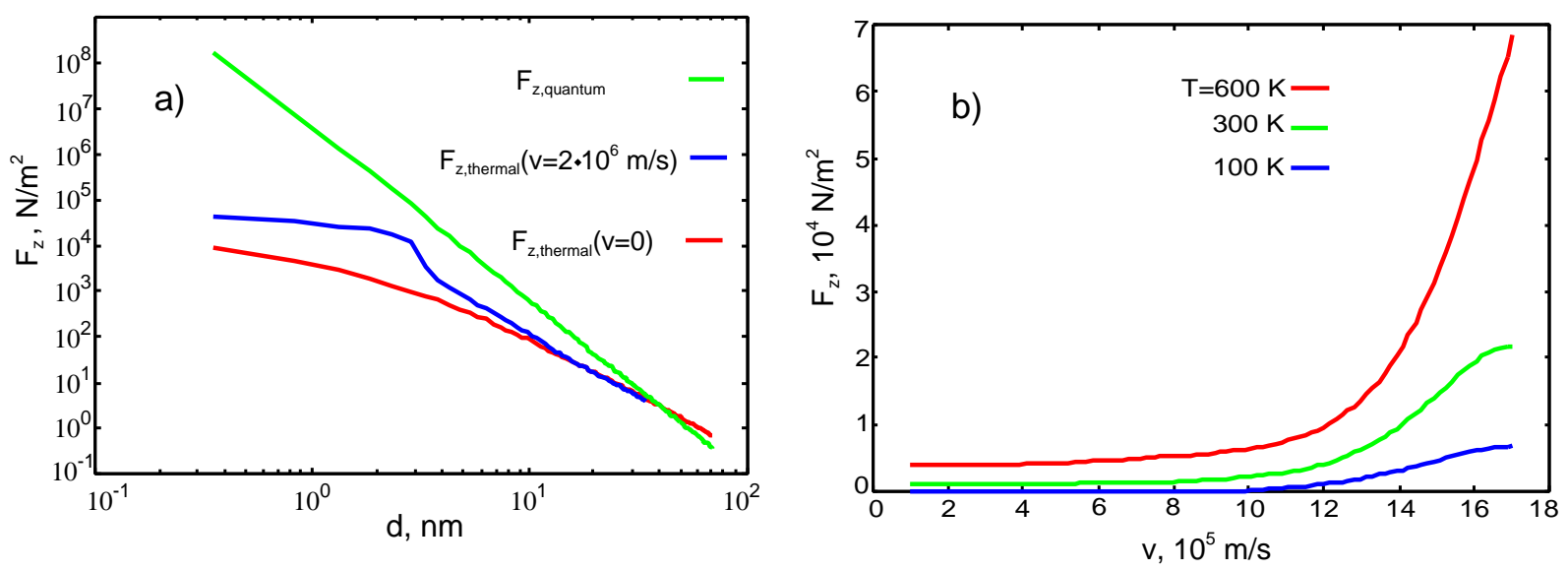

FIG. 1: The Casimir forces between two graphene sheets with carrier concentration $n=10^{16} \mathrm{~m}^{-2}$. (a) The dependence of the Casimir force on the separation $d$ between the sheets. The thermal and quantum contributions to the total Casimir force are shown separately. The thermal contribution is shown for $T=600 \mathrm{~K}$ and for the drift velocities $v=0$ and $v=2 \cdot 10^{6} \mathrm{~m} / \mathrm{s}$. (b) The dependence of the thermal Casimir force on the drift velocity of electrons $v$ in one of the graphene sheet at $d=1 \mathrm{~nm}$.

Eqs. (11) and (12) were initially obtained for $3 \mathrm{D}$-systems in Ref 31 at $T=0$ and in 39 ) for finite temperatures. However, in Ref. ( $(\underline{44})$ it was shown that the same formulas are valid for $2 \mathrm{D}$-systems. For $v<d k_{B} T / \hbar$ (at $d=1 \mathrm{~nm}$ and $T=300 \mathrm{~K}$ for $v<4 \cdot 10^{4} \mathrm{~m} / \mathrm{s}$ ) the main contribution to the friction (Eq. (11)) depends linearly on the sliding velocity $v$ so that the friction force $F_{x T}=\Gamma v$ where at $T_{1}=T_{2}=T$ the friction coefficient $\Gamma$ is given by

$$
\Gamma=\frac{\hbar^{2}}{8 \pi^{2} k_{B} T} \int_{0}^{\infty} \frac{d \omega}{\sinh ^{2}\left(\frac{\hbar \omega}{2 k_{B} T}\right)} \int_{0}^{\infty} d q q^{3} e^{-2 q d} \frac{\operatorname{Im} R_{1}(\omega) \operatorname{Im} R_{2}(\omega)}{\left|1-e^{-2 q d} R_{1}(\omega) R_{2}(\omega)\right|^{2}}
$$

Due to the presence of an exponential factor in the expression (3) for the thermal contribution to the Casimir force, the integration over frequency is effectively limited to $\omega<\omega_{T}=k_{B} T / \hbar$. Thus for $q_{x} v \sim v / d>\omega_{T}$ (at room temperature and for $d=1 \mathrm{~nm}$ this condition corresponds to the velocities $v>10^{5} \mathrm{~m} / \mathrm{s}$ ) the integrand will be modified in the whole range of integration, which will give rise to the significant change of the thermal Casimir force. This change will be especially large in the case of resonant photon tunneling when the integrand has sharp resonances. The integrand in the expression for the zero-temperature contribution to the Casimir force does not contain any sharp cut-off in the frequency integration. Thus the range of integration will be more wide and the change of the zero-temperature contribution will be significant only for much higher velocities then for the thermal contribution.

Fig. 1 1 a shows the dependence of the Casimir force between two graphene sheets on the separation $d$ between the sheets. The thermal and quantum contributions are shown separately. The thermal contribution was calculated for $T=600 \mathrm{~K}$ and for the drift velocities $v=0$ and $v=2 \cdot 10^{6} \mathrm{~m} / \mathrm{s}$. The thermal contribution becomes larger then the quantum contribution for $d>50 \mathrm{~nm}$. For $d<5 \mathrm{~nm}$ the thermal contribution calculated for $v=2 \cdot 10^{6}$ $\mathrm{m} / \mathrm{s}$ is significantly larger then the thermal contribution calculated at $v=0$. For example, at $d \approx 3 \mathrm{~nm}$ the drift motion of the electrons gives rise to the increase of the thermal Casimir force by one order of magnitude, and in this case the thermal contribution is only one order of magnitude smaller then the quantum contribution, and can be measured experimentally. Figure 1b shows the dependence of the thermal Casimir force $F_{z T}$ on the drift velocity of the electrons in the graphene sheet at $d=1 \mathrm{~nm}$. Note the significant change of the thermal Casimir force for $v / d>\omega_{T}$ (at room temperature and for $d=1 \mathrm{~nm}$ this condition corresponds to the velocities $v>10^{5} \mathrm{~m} / \mathrm{s}$ ). This change is connected with resonant photon tunneling. In this case the photon emitted by the moving electron system (with energy $\omega_{p h}(q)=q_{x} v-\omega_{e h}(q)$, where $\omega_{e h}(q)$ is the energy of the electron-hole pair excitation with momentum q, will create excitation with energy $\omega_{e h}(q)$ in other graphene sheet. In the case of graphene the energy of the electron-hole pair excitation $\omega_{e h}(q) \approx v_{F} q$, where $v_{F}$ is the Fermi velocity. Resonance occurs when $q_{x} v \approx 2 v_{F} q$, which corresponds to $v>2 v_{F} \approx 2 \cdot 10^{6} \mathrm{~m} / \mathrm{s}$, in accordance with the numerical calculations.

The Casimir friction force between two graphene sheets can be measured in frictional drag experiment. Such experiment was proposed theoretically some years ago $\underline{42,43}$ and performed experimentally for 2D-quantum wells 44,45 . In these experiments a current is driven through layer 1. Due to the proximity of the layers, the interlayer interactions will induce a current in layer $\mathbf{2}$ due to a friction stress acting on the electrons in the layer $\mathbf{2}$ from layer $\mathbf{1}$. If the layer $\mathbf{2}$ is an open circuit, an electric field $E_{1}$ will develop in the layer whose influence cancels the frictional stress $\sigma$ between 

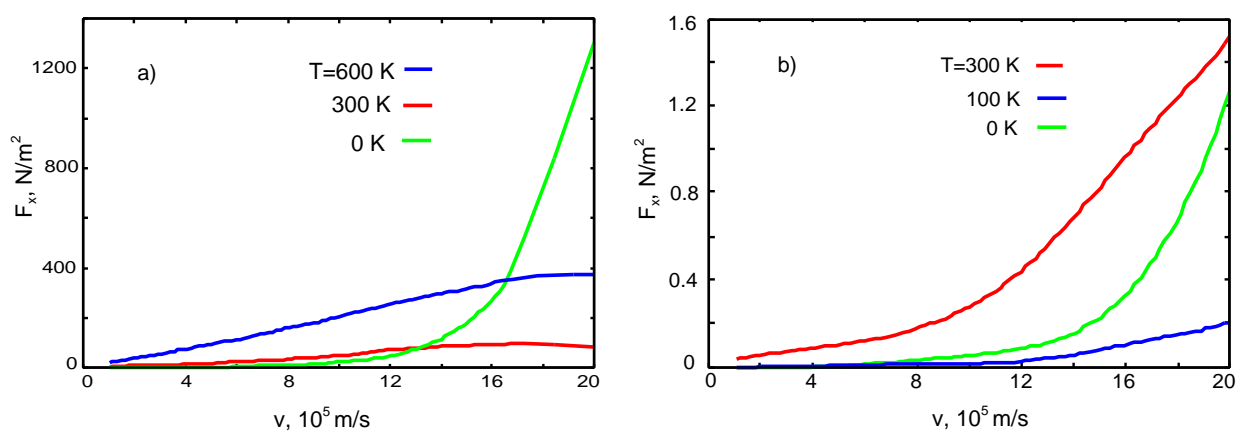

FIG. 2: The Casimir friction force between two graphene sheets at the carrier concentration $n=10^{12} \mathrm{~cm}^{-2}$. The finite temperature curves show only the thermal contributions to the friction. (a) Dependence of friction force between graphene sheets on the drift velocity of charge carriers in one graphene sheet at the layer separation $d=1 \mathrm{~nm}$. (b) The same as in (a) but at $d=10 \mathrm{~nm}$.

the layers. In one experiment $\underline{44}$ the drift velocity $v \sim 10^{2} \mathrm{~m} / \mathrm{s}$. According to the theory of the Casimir friction ${ }^{34,39}$, at such velocities the thermal fluctuation give the dominant contribution to the friction, and the theoretical predictions are in an agreement with experiment.

Frictional drag between graphene sheets was measured recently in Refs $\underline{46}, \underline{47}$. This study has fueled the recent theoretical investigations of frictional drag between graphene sheets mediated by a fluctuating Coulomb field $\underline{48-54}$. All these investigations assumes that the friction depends linear to the current density (or drift velocity $v$ of the charge carries). Thus only the thermal contribution to the frictional drag was included. In the linear approximation the electric field induced by the frictional drag depends linearly on the current density $J=n e v$ (or drift velocity $v$ of the charge carries), $E=\rho_{D} J=F_{x T} / n e=\Gamma J /(n e)^{2}$, where $\Gamma$ is the friction coefficient, $\rho_{D}=\Gamma /(n e)^{2}$ is the drag resistivity. For $\omega<v_{F} q$ and $q<2 k_{F}$ the dielectric function of graphene has the following form ${ }^{37}$

$$
\varepsilon_{0}(\omega, q) \approx 1+\frac{4 e^{2} k_{F}}{\hbar v_{F} q}\left(1+i \frac{\omega}{v_{F} q}\right)
$$

and the reflection amplitude

$$
R_{0}(\omega, q)=\frac{\varepsilon_{0}(\omega, q)-1}{\varepsilon_{0}(\omega, q)} \approx 1+i \frac{\hbar \omega}{4 e^{2} k_{F}},
$$

and Eqs.(15), (13) give the known resultt $\underline{48}$

$$
\rho_{D}=\frac{\Gamma}{(n e)^{2}}=\frac{h}{e^{2}} \frac{\pi \zeta(3)}{32}\left(\frac{k_{B} T}{\epsilon_{F}}\right)^{2} \frac{1}{\left(k_{F} d\right)^{2}} \frac{1}{\left(k_{T F} d\right)^{2}},
$$

where $k_{T F}=4 e^{2} k_{F} / \hbar v_{F}$ is the Thomas-Fermi screening wave vector. The frictional drag force is much higher for high drift velocities $\left(\sim 10^{6} \mathrm{~m} / \mathrm{s}\right)$, where it depends nonlinearly on the drift velocity, and is dominated by the quantum friction, existence of which was recently hot debated ${ }^{55} \underline{-60}$. For $v<v_{F}$ Eqs.(15), (12) give the following result for quantum friction

$$
F_{x 0}=\frac{\hbar v}{d^{4}} \frac{15 \zeta(5)}{128 \pi^{2}}\left(\frac{v}{v_{F}}\right)^{2} \frac{1}{\left(k_{T F} d\right)^{2}} .
$$

In linear approximation $E=5 \times 10^{-4} v$ (SI-units) for $T=300 \mathrm{~K}$ and $d=10 \mathrm{~nm}$. For a graphene sheet of length $1 \mu \mathrm{m}$, and with $v=100 \mathrm{~m} / \mathrm{s}$ this electric field will induce the voltage $V=10 \mathrm{nV}$.From Eqs. (16) and (17) the ratio of quantum and thermal friction $F_{x 0} / F_{x T}=F_{x 0} /(n e)^{2} \rho_{D} v \approx\left(15 / 8 \pi^{2}\right)\left(v / v_{T}\right)^{2}$, where $v_{T}=\omega_{T} d$. Thus, for $v>v_{T}$ the friction is dominated by quantum friction (at $d=1 \mathrm{~nm}$ and room temperature: $v_{T} \approx 4 \cdot 10^{4} \mathrm{~m} / \mathrm{s}$ ),

Figures $2 \mathrm{a}$ and $2 \mathrm{~b}$ show that much larger electric fields can be induced at $d=1 \mathrm{~nm}$ (a) and $d=10 \mathrm{~nm}(\mathrm{~b})$ at large velocities. In these figures the contributions to friction from thermal and quantum fluctuations are shown separately, where the friction force is related to the electric field: $F_{x}=n e E$. For $v<10^{5} \mathrm{~m} / \mathrm{s}$ the frictional drag effect for the graphene sheets strongly depends on temperature, i.e. it is determined mainly by the thermal fluctuations. However, for $v>10^{6} \mathrm{~m} / \mathrm{s}$ it will be dominated by quantum fluctuations. Strong enhancement of friction occurs in the case of resonant photon tunneling. As discussed above, resonant photon tunneling occurs for $v>2 v_{F} \approx 2 \cdot 10^{6} \mathrm{~m} / \mathrm{s}$. For 
such velocities and $d=1 \mathrm{~nm}$ quantum friction dominates over the thermal contribution even at room temperature (see Fig:1 ). For $d=10 \mathrm{~nm}$ quantum friction dominates at low temperatures (see Fig, $2 \mathrm{~b}$ ). As discussed in Ref. $\underline{41}$ quantum friction can be also detected by measuring the transport properties of non-suspended graphene on a $\mathrm{SiO}_{2}$ substrate.

Concluding remarks.-We have calculated the dependence of the thermal Casimir force between two graphene sheets on the drift velocity of the charge carriers in one graphene sheet. We have found that the drift motion of the charge carriers in graphene produces changes in the thermal Casimir force which can be measured experimentally. The thermal Casimir force, as well as the Casimir friction force, are strongly enhanced in the case of resonant phonon tunneling. For resonant photon tunneling and small $d$, even for temperatures above room temperature, the Casimir friction is dominated by quantum friction due to quantum fluctuations. Quantum friction can be detected in friction drag experiment between graphene sheets. Another way to detect quantum friction consists in measuring of the transport properties of nonsuspended graphene on an $\mathrm{SiO}_{2}$ substrate in the high electric field.

\section{Acknowledgment}

A.I.V. acknowledges financial support from Russian Foundation for Basic Research (Grant N 12-02-00061-a) and European Science Foundation within activity "New Trends and Applications of the Casimir Effect". A.I.V. also thanks the Condensed Matter group of ICTP for hospitality during the time of working on this article.

1 H.B.G.Casimir, Proc. K. Ned. Akad. Wet., 51, 793 (1948).

2 E.M.Lifshitz, Zh. Eksp. Teor. Fiz., 29, (1955) [Sov. Phys. JETP 2,73 (1956)]

3 S. M. Rytov, Theory of Electrical Fluctuation and Thermal Radiation (Academy of Science of USSR Publishing, Moscow, 1953)

4 M. L. Levin and S. M. Rytov, Theory of eqilibrium thermal fluctuations in electrodynamics (Science Publishing, Moscow, 1967)

5 S. M. Rytov, Y. A. Kravtsov, and V. I. Tatarskii, Principles of Statistical Radiophyics(Springer, New York.1989), Vol.3

6 I.E.Dzyaloshinskii, E.M.Lifshitz and L.P.Pitaevskii, Adv.Phys. 10, 165 (1961)

7 S.K.Lamoreaux, Phys.Today 60, 40 (Februrary,2007)

8 P.W.Milloni, The Quantum Vacuum: An Introduction to Quantum Electrodynamics (Academic,1993)

9 A.O.Sushkov, W.J.Kim, D.A.R.Dalvit and S.K.Lamoreaux, Nature Phys. 7, 230 (2011).

10 F.M.Serry, D.Walliser and G.J.Maclay, J. Appl. Phys. 84, 2501 (1998).

11 E.Buks and M.L.Roukes, Phys.Rev.B 63, 033402 (2001).

12 G.L.Klimchitskaya, U.Mohideen and V.M.Mostepanenko, Rev. Mod. Phys. 81, 1827 (2007).

13 J.N.Munday, F.Capasso and V.A.Parsegian, Nature (London) 457, 170 (2007).

14 A.O.Sushkov, W.J.Kim, D.A.R.Dalvit and S.K.Lamoreaux, Phys. Rev. Lett. 107, 171101 (2011).

15 Y.Bao, R.Guérout, J.Lussange, A.Lambrecht, R.A. Cirelli, F.Klemens, W.M.Mansfield, C.S.Pai and H.B.Chan, Phys. Rev. Lett. 105, 250402 (2010).

16 A.W.Rodriguez, W.J.Kim, F.Capasso and S.G.Johnson, Nature Photon. 5, 211 (2011).

17 R.Zhao, J.Zhou, Th.Koschny, E.N.Economou and C.M.Soukoulis, Phys. Rev. Lett. 103, 103602 (2009).

18 M.Bordag, B.Geyer, G.L.Klimchitskaya and V.M.Mostepanenko, Phys.Rev.B 74, 205431 (2006).

19 M.Bordag, I.V.Fialkovsky, D.M.Gitman and D.V.Vassilevich, Phys.Rev.B 80, 245406 (2009).

20 D.Drosdoff and L.M.Woods, Phys.Rev.A 84, 062501 (2011).

21 D.Drosdoff and L.M.Woods, Phys.Rev.B 82, 155459 (2010).

22 I.V.Fialkovsky, V.N.Marachevsky and D.V.Vassilevich, Phys.Rev.B 84, 035446 (2011).

23 B.E.Sernelius, EPL 95, 57003 (2011).

24 V.Svetovoy, Z.Moktadir, M.Elwenspoek and H.Mizuta, EPL 96, 14006 (2011).

25 J.Sarabadani, A.Naji, R.Asgari and R.Podgornik, Phys.Rev.B 84, 155407 (2011).

26 G.Gómez-Santos, Phys.Rev.B 80, 245424 (2009).

27 K.S. Novoselov, A.K. Geim, S.V. Morosov, D. Jiang, Y. Zhang, S.V. Dubonos, I.V. Grigorieva, and A.A. Firsov, Science 306, 666 (2004).

${ }^{28}$ K.S. Novoselov, A.K. Geim, S.V. Morosov, M.I. Katsnelson, I.V. Grigorieva, S.V. Dubonos, and A.A. Firsov, Nature (London) 438, 197 (2005).

29 A.K. Geim and K.S. Novoselov, Nat. Mater. 6, 183 (2007).

30 Geim A K Science 3241530 (2009)

31 J.B. Pendry, J. Phys.C, 9, 10301 (1997).

32 B. Shapiro, Phys. Rev. B 82, 075205 (2010)

33 A.I.Volokitin and B.N.J. Persson, Phys. Rev. B 78, 155437 (2008); ibid. 81, 239901(E) (2010).

34 A.I.Volokitin and B.N.J. Persson, J.Phys.: Condens. Matter 13, 859 (2001). 
35 N.D.Mermin, Phys. Rev. B 1, 2362 (1970).

36 B. Wunscvh, T. Stauber, F. Sols, and F. Guinea, New J.Phys. 8,318 (2006).

37 E.H. Hwang, S.Das Sarma, Phys. Rev. B 75, 205418 (2007).

38 J.H. Chen, C. Jang, S. Xiao, M. Ishigami and M.S.Fuhrer, Nat. Nanotechnol. 3, 206 (2008)

39 A.I.Volokitin and B.N.J. Persson, J.Phys.: Condens. Matter 11, 345 (1999); Phys.Low-Dim.Struct. 7 /8, 17 (1998).

40 A.I.Volokitin and B.N.J. Persson, Rev. Mod. Phys. 79, 1291 (2007).

41 A.I. Volokitin and B.N.J. Persson, Phys. Rev. Lett.106, 094502 (2011).

42 M.B. Pogrebinskii, Fiz.Tekh.Poluprov. 11, 637 (1977) [Sov.Phys. Semicond. 11, 372 (1977)].

43 P. J. Price, Physica B+C 117,750 (1983).

44 T.J. Gramila, J.P. Eisenstein, A.H. MacDonald, L.N. Pfeiffer, and K. W. West, Phys. Rev. Lett. 66, 1216 (1991).

45 U. Sivan, P.M. Solomon, and H. Shtrikman, Phys. Rev. Lett. 68, 1196 (1992).

46 S.Kim, I.Jo, J.Nah, Z.Yao, S.K.Banerjee and E.Tutuc, Phys. Rev. B 83161401 (2011).

47 R.V.Gorbachev, A.K.Geim, M.I.Katsnelson, K.S.Novoselov, T.Tudorovskyiy, T.V.Grigorieva, A.H.MacDonald, K.Watanabe, T.Taniguchi and L.P.Ponamarenko Nature Phys. 8896 (2012).

48 W.K.Tse, BenYu-Kuang.Hu and S.DasSarma, Phys. Rev.B 76081401 (2007).

49 M.I.Katsnelson, Phys. Rev.B 84 041407(R) (2011).

50 N.M.R.Peres, J.M.R.Lopes des Santos and A.H.Castro Neto, Europhys. Lett 9518001 (2011).

51 E.H.Hwang, R.Sensarma and S.DasSarma, Phys. Rev. B 84245441 (2011).

52 B.N.Narozhny, M.Titov, I.V.Gornyi and P.M.Ostrovsky, Phys. Rev. B 85195421 (2012).

53 M.Carrega, T.Tudorovskiy, A.Principi, M.I.Katsnelson and M.Polini M 2012 New J. Phys. 14063033 (2012).

54 B.Amorin and N.M.R.Peres, J. Phys.:Condens. Matter. 24335602 (2012).

55 T.G. Philbin and U. Leonhardt, New J. Phys. 11,033035 (2009).

56 J.B. Pendry, New J. Phys. 12, 033028 (2010).

57 U. Leonhardt, New J. Phys. 12,068001 (2010).

58 J.B. Pendry, New J. Phys. 12, 068002 (2010).

59 A.I.Volokitin and B.N.J. Persson, New J. Phys. 13, 068001 (2011).

60 T.G.Philbin, and U.Leonhardt, New J. Phys. 13, 068002 (2011). 